\title{
THE RATIO OF TOTAL TO SELECTIVE EXTINCTION FROM THE CLUSTER DIAMETER METHOD
}

\author{
DA NIEL H. HARRIS
}

Steward Observatory and Institute of Atmospheric Physics, University of Arizona, Ariz., U.S.A.

\begin{abstract}
One hundred and fifty six open clusters with modern photometry, and apparent diameter measures by Trumpler, have been used to measure the mean ratio of total to selective extinction, $\bar{R}$. It is found that cluster diameter is related to both concentration class and richness class. Diameter also seems to depend on the background stellar density and to decrease with reddening. With such effects measured and removed $\bar{R}=3.15 \pm 0.20$. This result is not significantly dependent on the cluster's position in space.
\end{abstract}

Of the methods available for measuring the interstellar extinction, only the cluster diameter method can measure the grey extinction of large particles. This method is therefore potentially very useful. To fully realize this potential we must collect accurate diameter measures and photometry for a large homogeneous sample of open clusters.

Trumpler (1930) and Wallenquist (1959) have published the only large and uniform sets of open cluster diameter measures. I have made an effort to assemble the necessary photometry of these clusters. Photometric data tabulated in Becker (1963), Johnson et al. (1961), and Hagen (1970) have been examined and inter-compared. Where possible the cluster color-magnitude diagrams were studied. Only 156 of the clusters had well determined distance modulii and color excesses. These were transformed to the UBV system. The photometric data were then used to compute the cluster distances for various assumed values of the ratio of total to selective absorption, $\mathbf{R}$. For the purposes of this paper we consider clusters with $E_{B-V}<0.15$ to be unreddened. Such small color excesses with normal values of $\mathbf{R}$ change the distance modulus by less than one-half magnitude.

Figures 1,2, 3, and 4 present the log distance-log apparent angular diameter data for Trumpler's diameters. It is evident that the reddened and unreddened clusters follow nearly the same distance-apparent diameter relation. This is confirmed by the near agreement of the last squares fits also shown. Note that with the large scatter in apparent diameter the general distribution of points doesn't change rapidly with R. The best agreement of the reddened and unreddened clusters seems yo be near $\mathbf{R}=3.2$.

The distance-apparent diameter plots for Wallenquist's diameters look nearly the same as the Trumpler plots except that there are fewer points. Figure 5 presents the distance-apparent diameter data for Wallenquist's diameters and $\mathbf{R}=3.2$.

There are two noteworthy peculiarities in the distance-apparent diameter relations. First, the steep slope and noticable curvature of the least squares fits suggest that effects are present other than simply the reduction of apparent size with distance. 


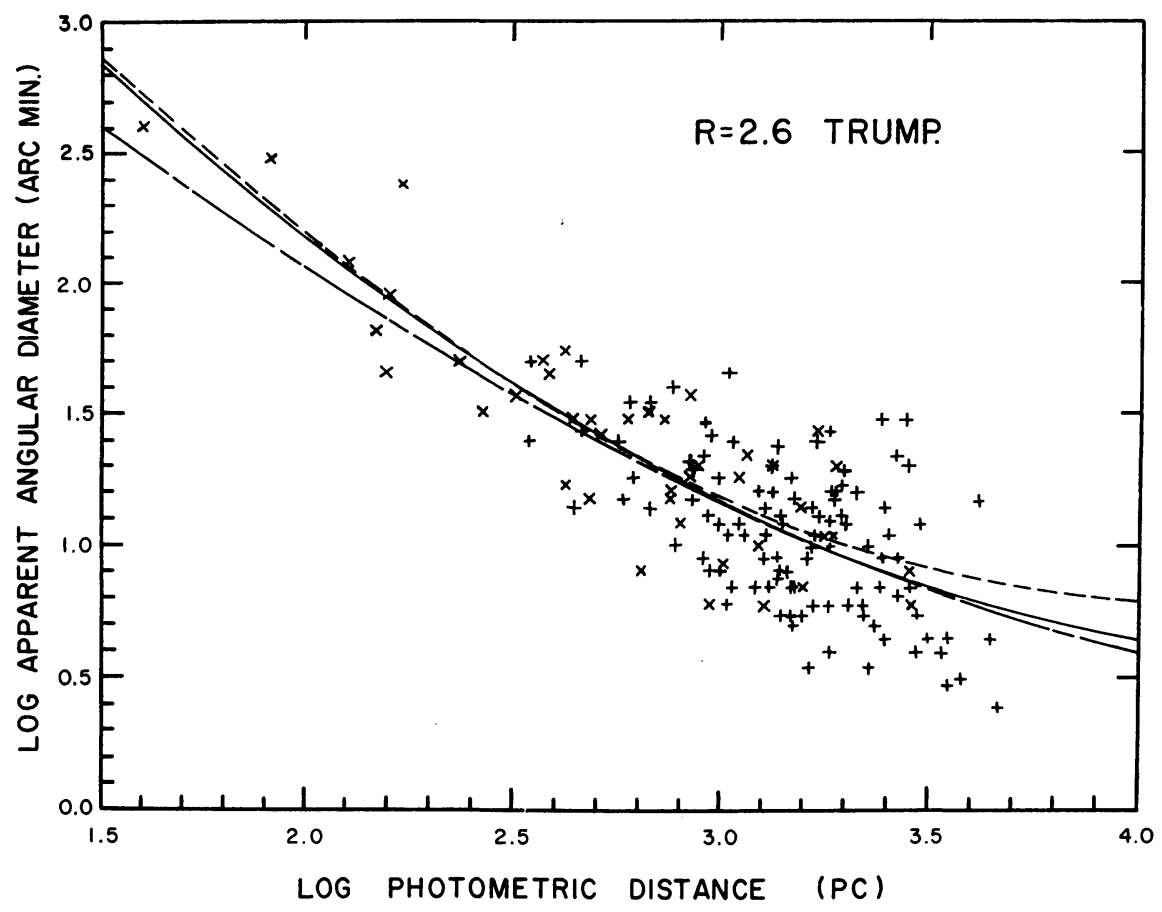

Fig. 1. The apparent angular diameter-photometric distance relations for open clusters with Trumpler diameters and $R=2.6$. The $(\times)$ points are clusters with $E_{B-V}<0.15$ and the (+) points are clusters with $E_{B-V} \geq 0.15$. The curves are least squares fits to the points. The dashed curve fits the $(\times)$ points. The solid-dashed curve fits the $(+)$ points. The solid curve fits all the points.

Second, the clusters appear to shrink with increased color excess. This is shown by the difference between the fits to reddened and unreddened clusters. However, if we assume $\mathbf{R} \simeq 2.6$ then there is no shrinkage at all. The measurement of the apparent shrinkage with $E_{B-V}$ can not be separated from the problem of finding the best value of $\mathbf{R}$. Before we can understand these effects we must attempt to reduce the size of the diameter residuals. In order to do this we will search for dependences of the apparent diameters on other variables, then remove these dependences.

We should expect to find a dependence of apparent diameter on cluster type (Lynds, 1967). To test for such a dependence I have computed residuals in the logarithm of the apparent angular diameter relative to the least squares fits to all clusters regardless of color excess. These residuals show that Trumpler's diameters depend significantly on both concentration class and richness class. Wallenquist's diameters in contrast show no significant dependence on concentration. In order to correct for the type dependence in Trumpler's diameters I have computed mean type residuals. Residuals for all clusters of a particular concentration class and richness class have been averaged, except for those clusters that markedly deviate from the mean of their type.

Table I presents the preliminary mean type residuals for assumed $\mathbf{R}$ values of 2.8 , 


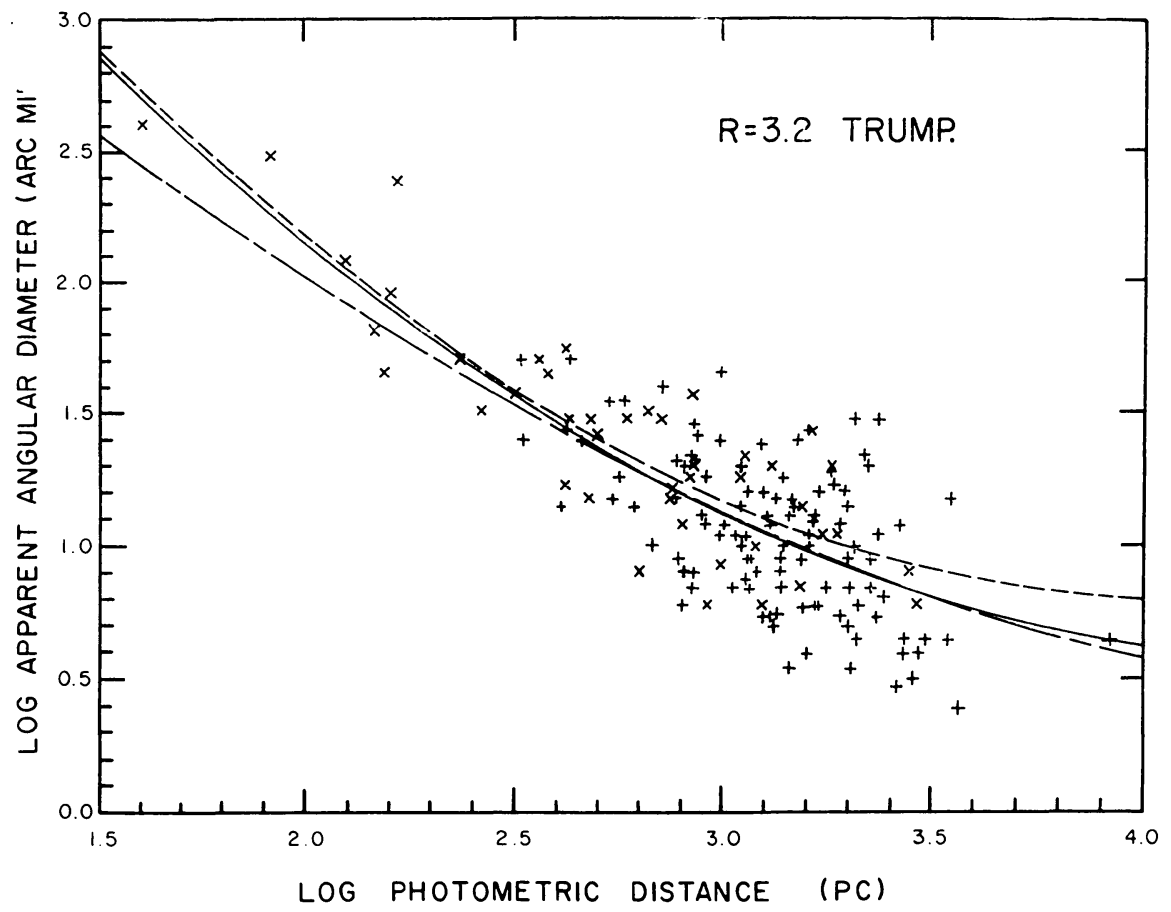

Fig. 2. The apparent diameter-distance relations for $R=3.2$.

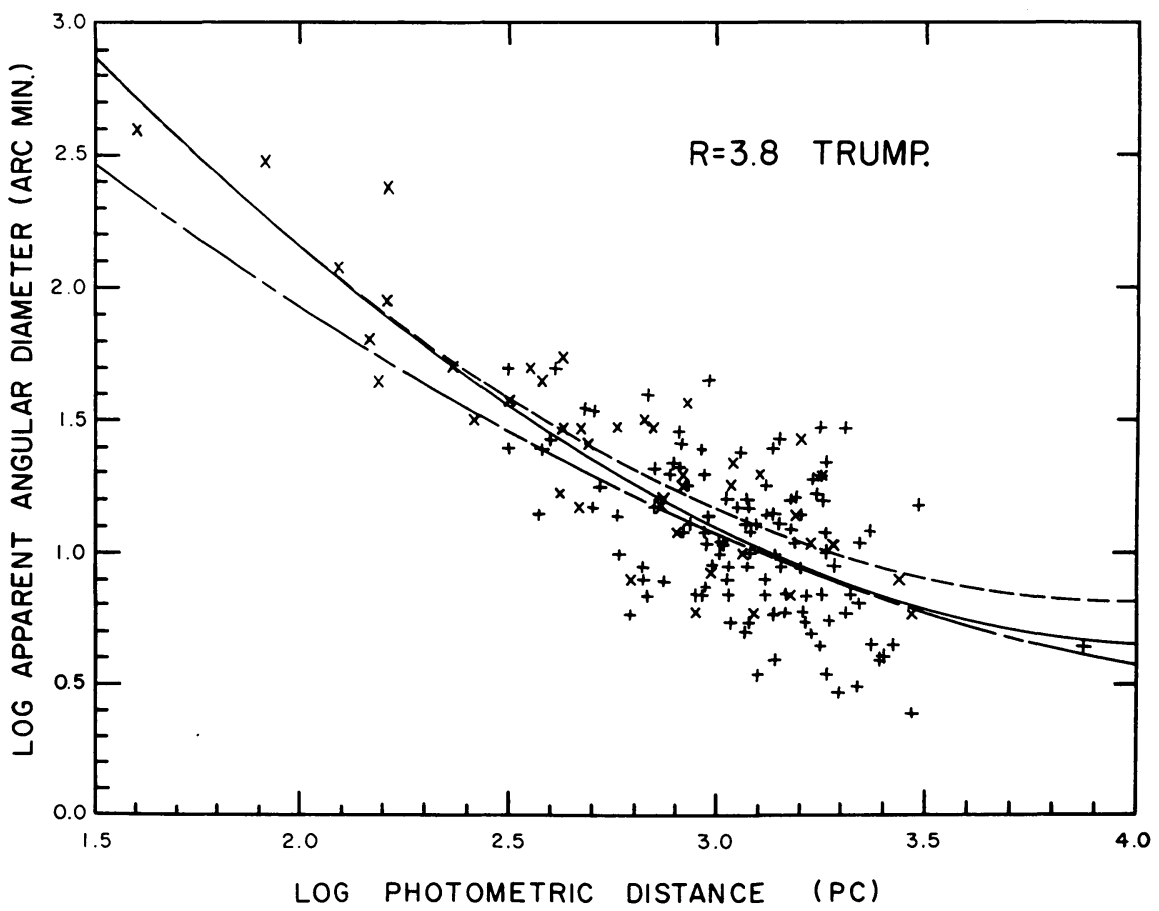

Fig. 3. The apparent diameter-distance relations for $R=3.8$. 


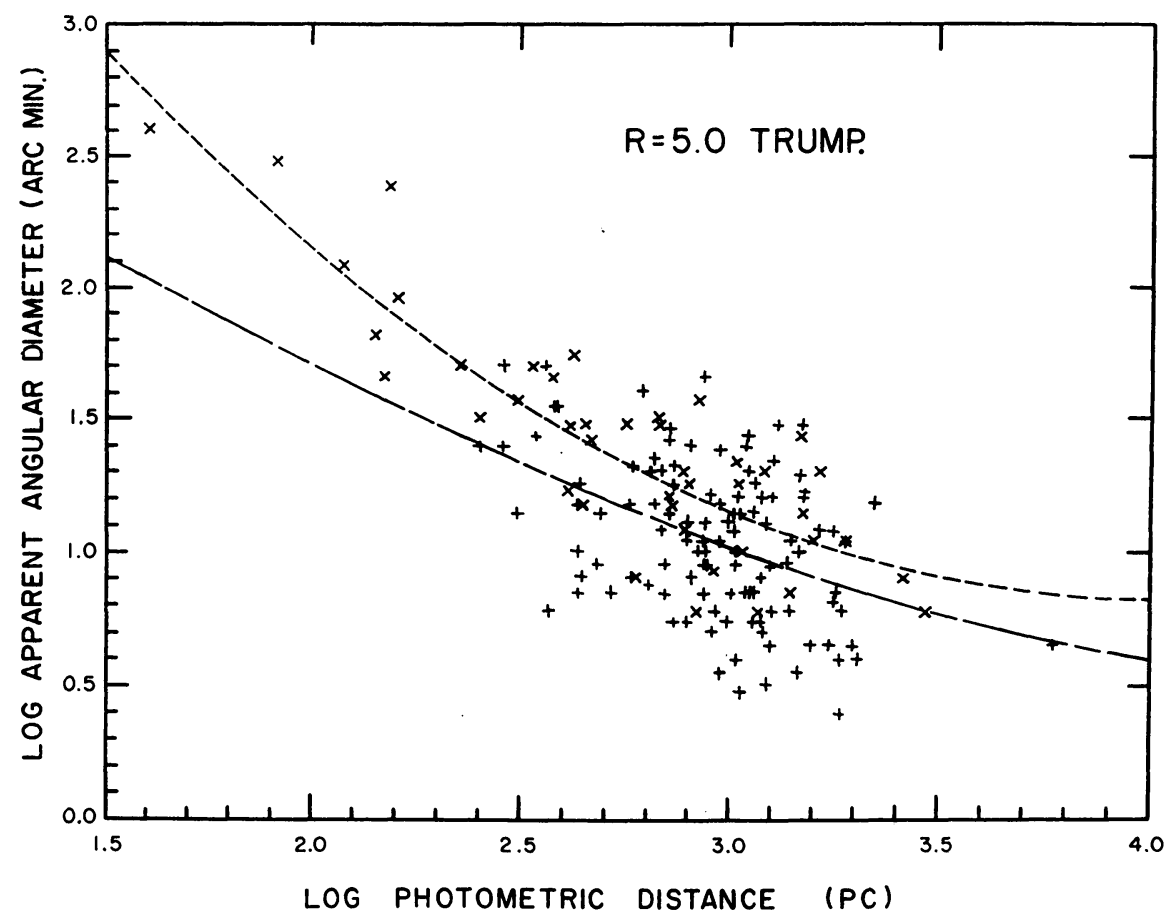

Fig. 4. The apparent diameter-distance relations for $R=5.0$.

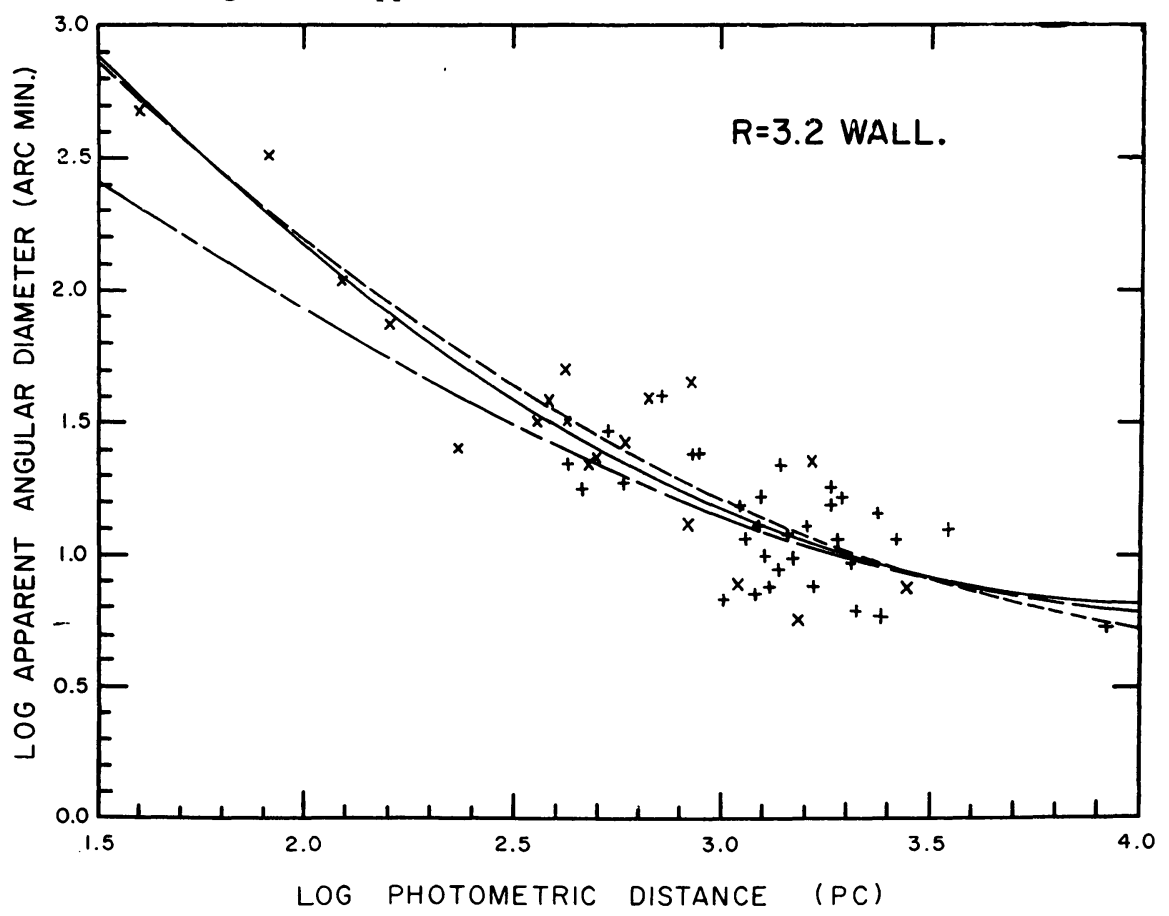

Fig. 5. The relation between apparent angular diameter and photometric distance for Wallenquist diameters and $R=3.2$. 
TABLE I

Preliminary mean type residuals to the logarithm of the angular diameter

\begin{tabular}{lllll}
\hline \multicolumn{5}{c}{$R=2.8$} \\
\hline & I & II & III & IV \\
\hline$r$ & +0.130 & +0.130 & +0.265 & +0.44 \\
$m$ & -0.07 & -0.02 & +0.265 & +0.29 \\
$p$ & -0.185 & -0.185 & 0.0 & +0.15 \\
\hline \multicolumn{5}{c}{$R=3.2$} \\
\hline & I & II & III & IV \\
\hline$r$ & +0.135 & +0.135 & +0.275 & +0.43 \\
$m$ & -0.07 & -0.02 & +0.275 & +0.27 \\
$p$ & -0.185 & -0.185 & 0.0 & +0.15 \\
\hline & \multicolumn{5}{c}{ II } \\
- & \multicolumn{5}{c}{$R=3.6$} & III & IV \\
\hline & I & II & +0.41 \\
$r$ & +0.140 & +0.140 & +0.285 & +0.25 \\
$m$ & -0.07 & -0.02 & +0.285 & +0.25 \\
$p$ & -0.185 & -0.185 & 0.0 & +0.15 \\
\hline
\end{tabular}

3.2 and 3.6. Because certain cluster types differ in mean $E_{B-V}$ from the mean $E_{B-V}$ of all the clusters, their mean type residuals contain terms like $C \cdot \Delta E_{B-V}$, where $\Delta E_{B-V}$ is this color excess difference. If we assume in turn that each value of $\mathbf{R}$ is correct then we may estimate the shrinkage coefficient $\mathbf{C}$, by either the separation of the least squares fits or by the deviation of the mean slope of the distance-apparent diameter relation from minus one. The first method is inaccurate because of the large scatter in the diameter measures. I have therefore corrected the mean type residuals using the slope deviation method. The corrections included in Table I are generally less than ten percent of the mean type residual.

Figures 6, 7 and 8 present the distance-apparent diameter data corrected for dependence on Trumpler type. New least squares fits are also shown. Note that the curvature in the new fits is slightly diminished while the apparent shrinkage with color excess is increased. The best agreement between the reddened and unreddened clusters will seems to be near $\mathbf{R}=3.2$.

With the type dependence removed the Trumpler diameters have noticably smaller residuals. In fact, the revised Trumpler diameters have smaller residuals than the Wallenquist diameters. The larger residuals of the Wallenquist diameters and their smaller number makes them unsuited for further study here.

The remaining Trumpler diameter residuals are still large compared to the residuals which might come from errors in the distance modulii. Therefore we need to search further for diameter dependences.

Figure 9 presents the residuals in the type corrected Trumpler diameters plotted 


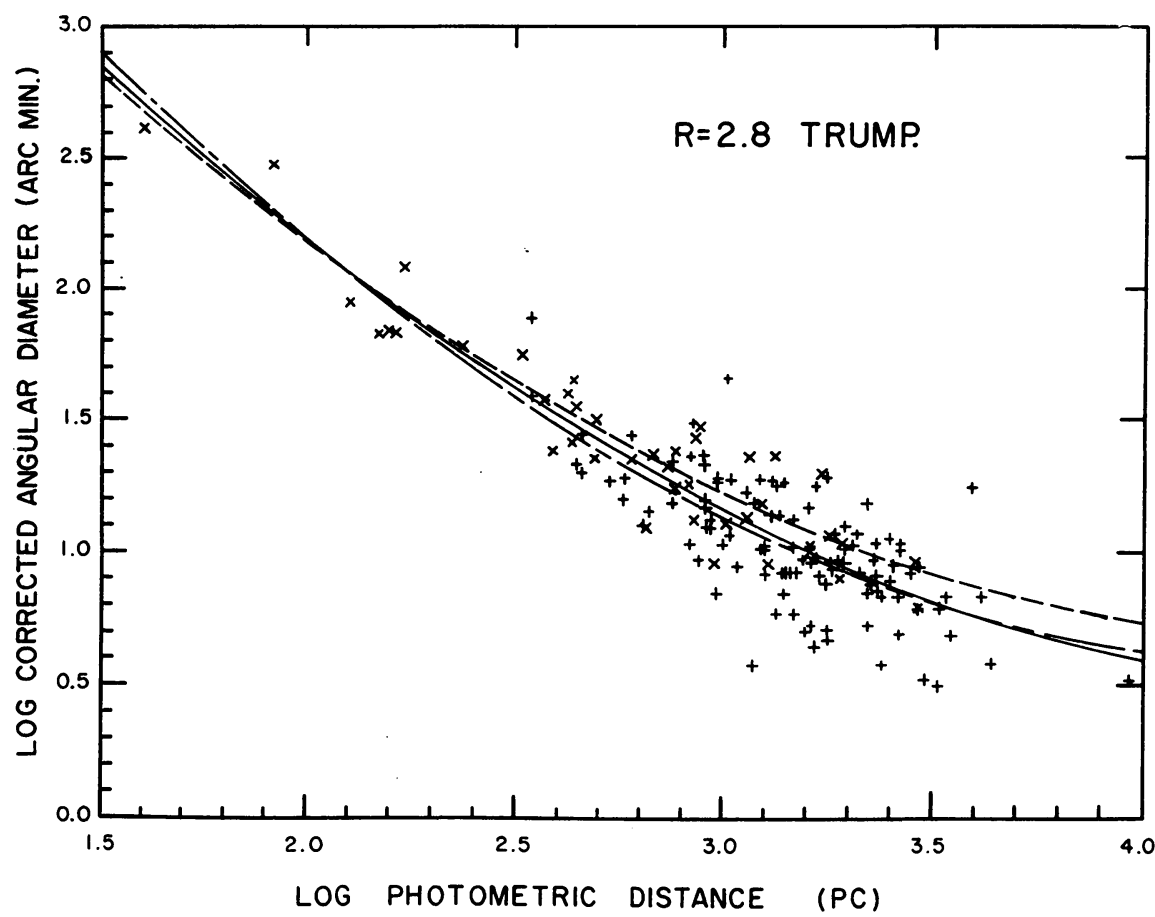

Fig. 6. The type corrected angular diameter-photometric distance relations for $R=2.8$.

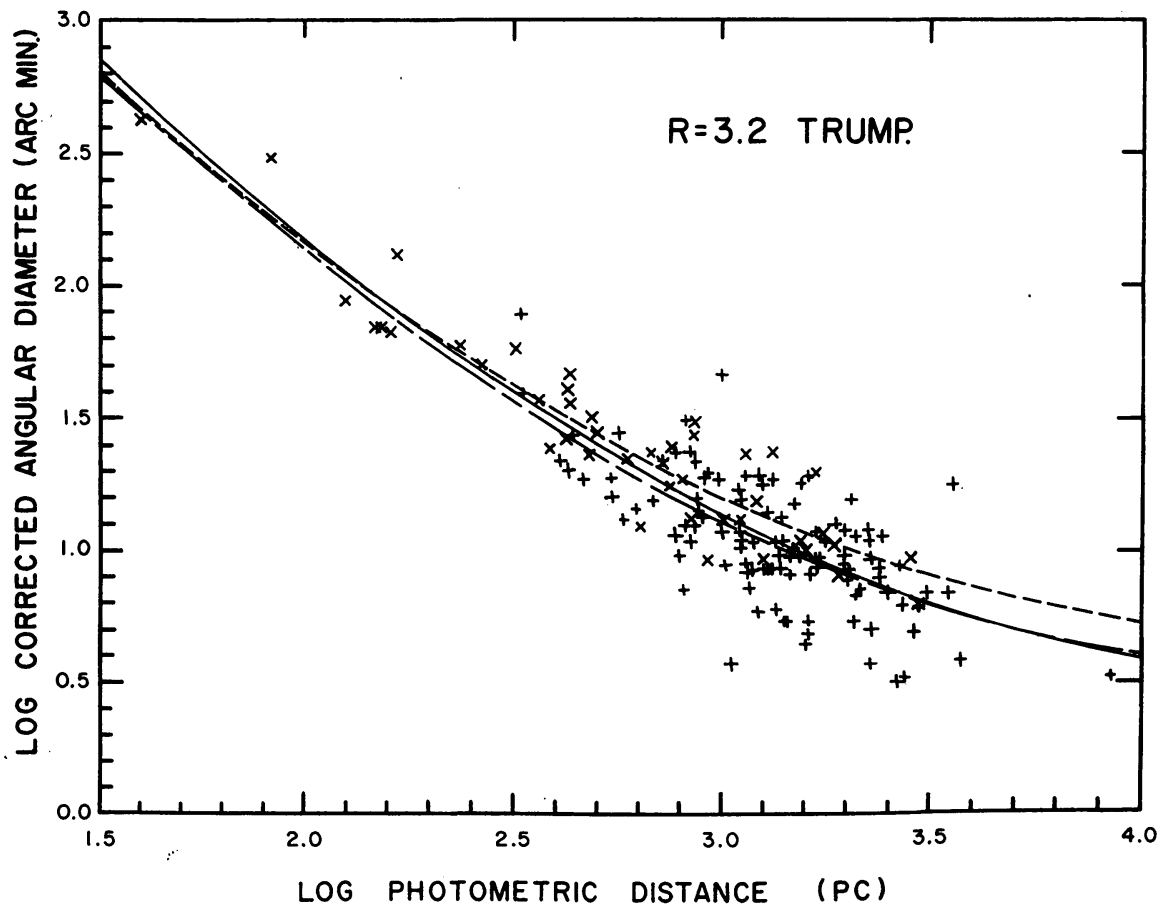

Fig. 7. The corrected angular diameter-distance relations for $R=3.2$. 


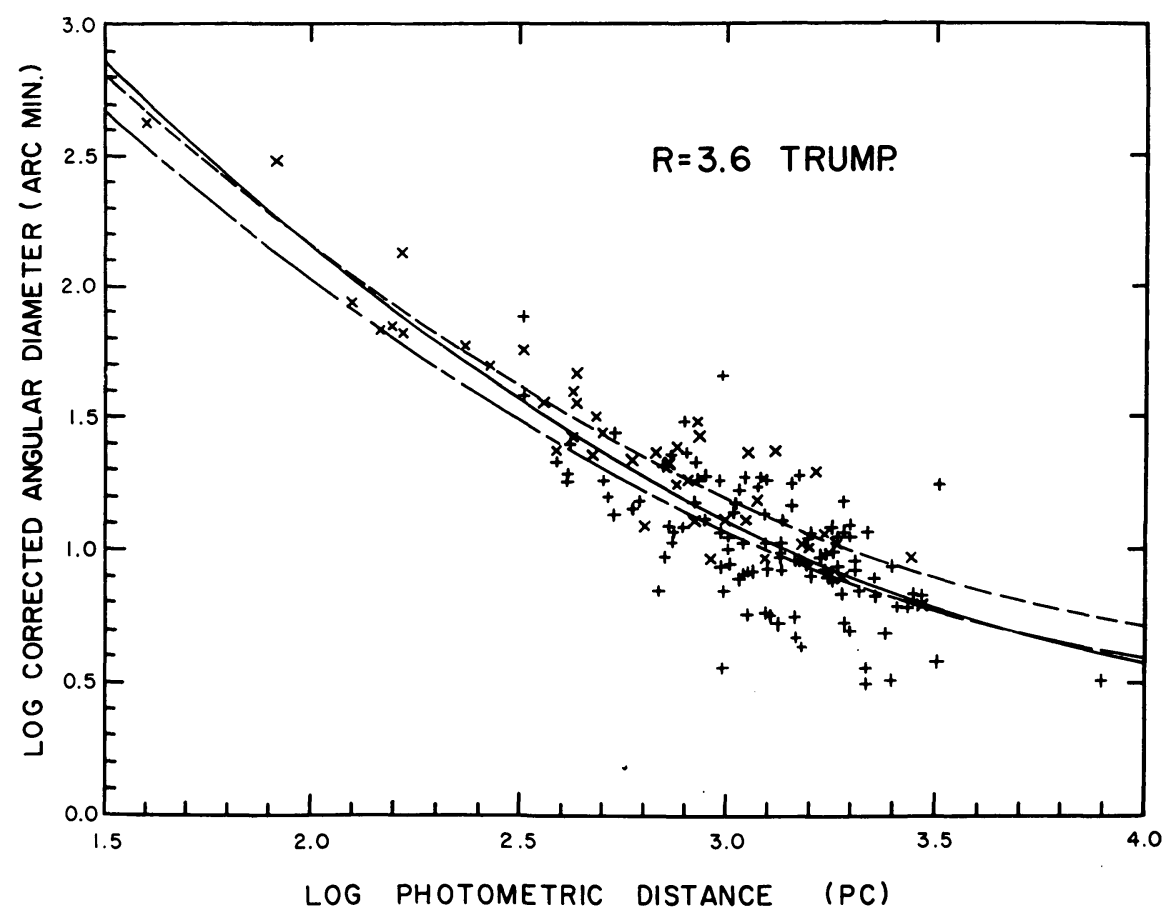

Fig. 8. The corrected angular diameter-distance relations for $R=3.6$.

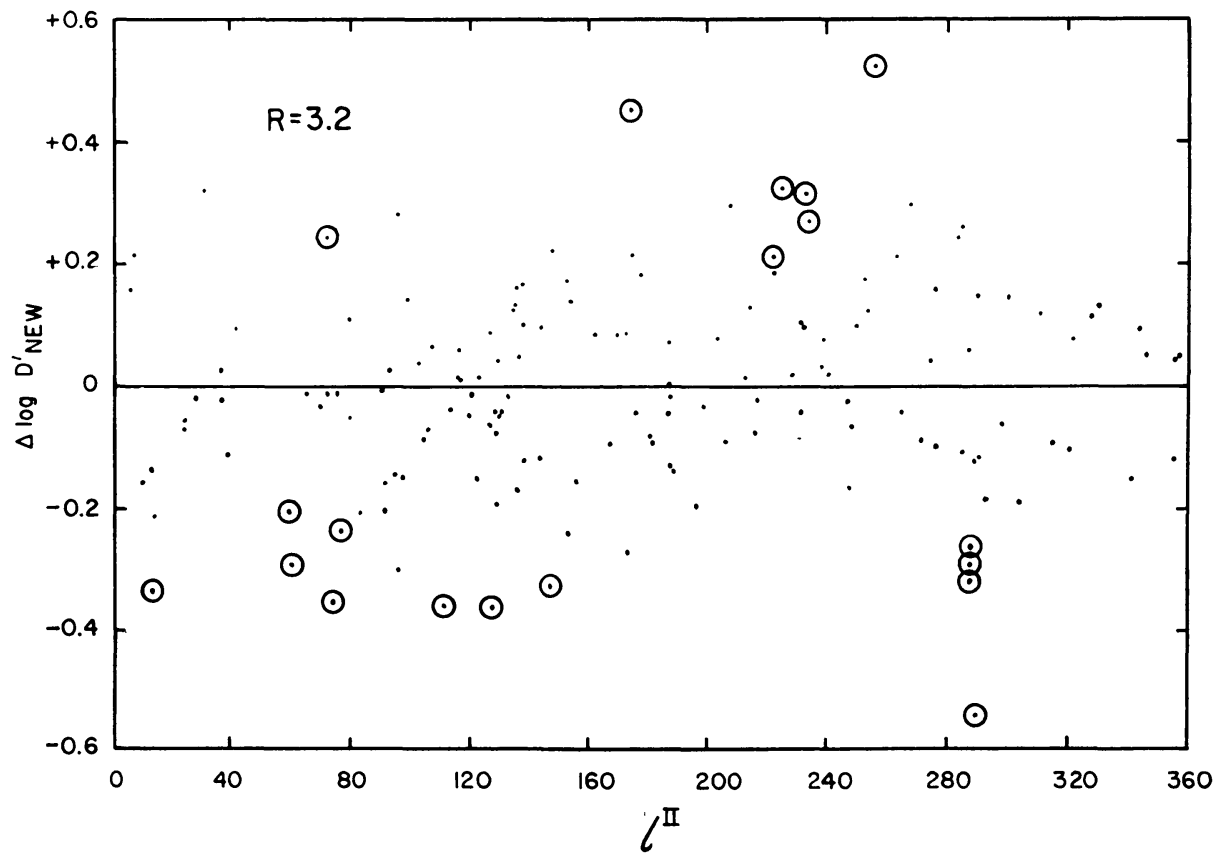

Fig. 9. The residuals in the type corrected angular diameters against longitude for $R=3.2$. The circled points were not included in the mean type residual computations. 
against galactic longitude. As the residuals do not vary rapidly with $\mathbf{R}$, I have assumed $\mathbf{R}=3.2$. The circled points represent clusters which were not included in the computation of the mean type residuals. It is clear that there is no large systematic longitude variation of cluster diameter. Further analysis of the revised Trumpler diameters shows no significant variation with distance from the galactic center, spiral arm number or spectrum of earliest member star. Combinations of these variable also yield no significant relations.

It may seem that we have exhausted the variables which might relate to the cluster diameter. But we have as yet considered only variables which might relate to the true diameter of the cluster. There may be effects which only appear to make the clusters change size. For example, if the diameter measures are made from material which does not have a sufficiently faint and uniform limiting magnitude or if the diameter measures are made for stars of variable brightness ratio fainter than the brightest members, then there will be a limiting magnitude effect. The care taken by Trumpler with his diameter measures probably precludes any significant limiting magnitude effect.

If we look at clusters of increasing distance, the cluster stars generally become fainter relative to the field stars. The cluster stars therefore appear less numerous relative

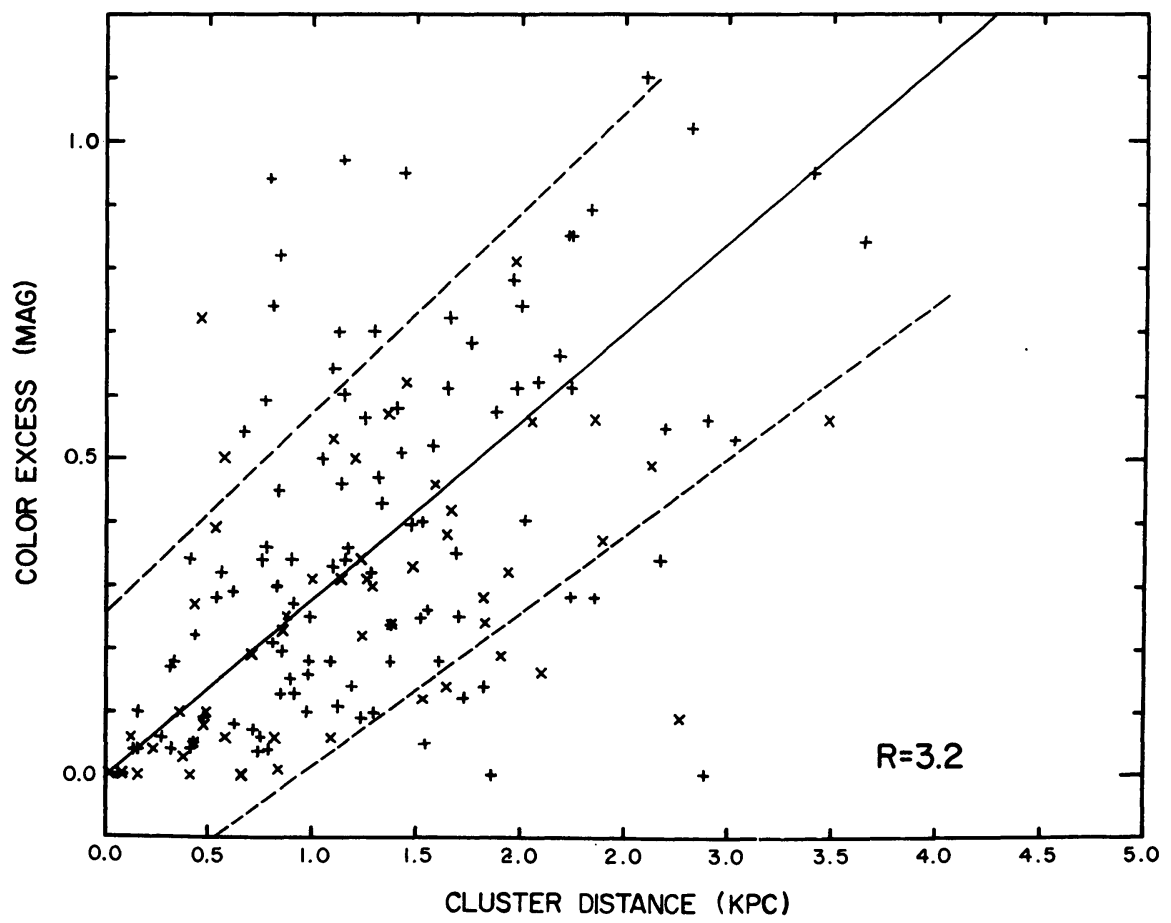

Fig. 10. The color excess, $E_{B-V}$ as a function of distance for $\mathbf{R}=3.2$. The $(\times)$ points are clusters with both Wallenquist and Trumpler diameters. The $(+)$ points are clusters with only Trumpler diameters. 
to the field stars of the same apparent magnitude. As the fainter cluster members are generally spread over a larger area than the brighter ones, the radius at which the cluster stars appear significant decreases with distance (Trumpler, 1922). If the cluster is at a large enough distance then the brighter field stars are ignored. Thus there is a distance beyond which the diameter is not further affected. This effect may be present in Figures 6, 7 and 8 for clusters with $\log r<2.8$.

Consider the influence of variations in the background star density. Near the cluster boundary the number density of stars falls off slowly. As a consequence, slight variations in the background may strongly influence the apparent cluster diameter. Trumpler has given a cluster density profile from an average of several clusters (Trumpler, 1922). The boundary slope is about five to one normalized to radius of one and central density of one. The background density is about one-quarter the central density. With such a boundary slope and background density a fractional change in the background density produces a two to three times larger fractional change in the apparent diameter. This effect may explain the increasing size of the inferred linear diameters of clusters beyond $\log r=2.8$.

These effects on the apparent diameters undoubtedly contribute to the residuals. But, as they are not well understood, nor are there adequate supplementary data available (e.g., background star densities), they can not be removed. Even unknown effects may be present. For expediency I will assume that the mean distance-apparent

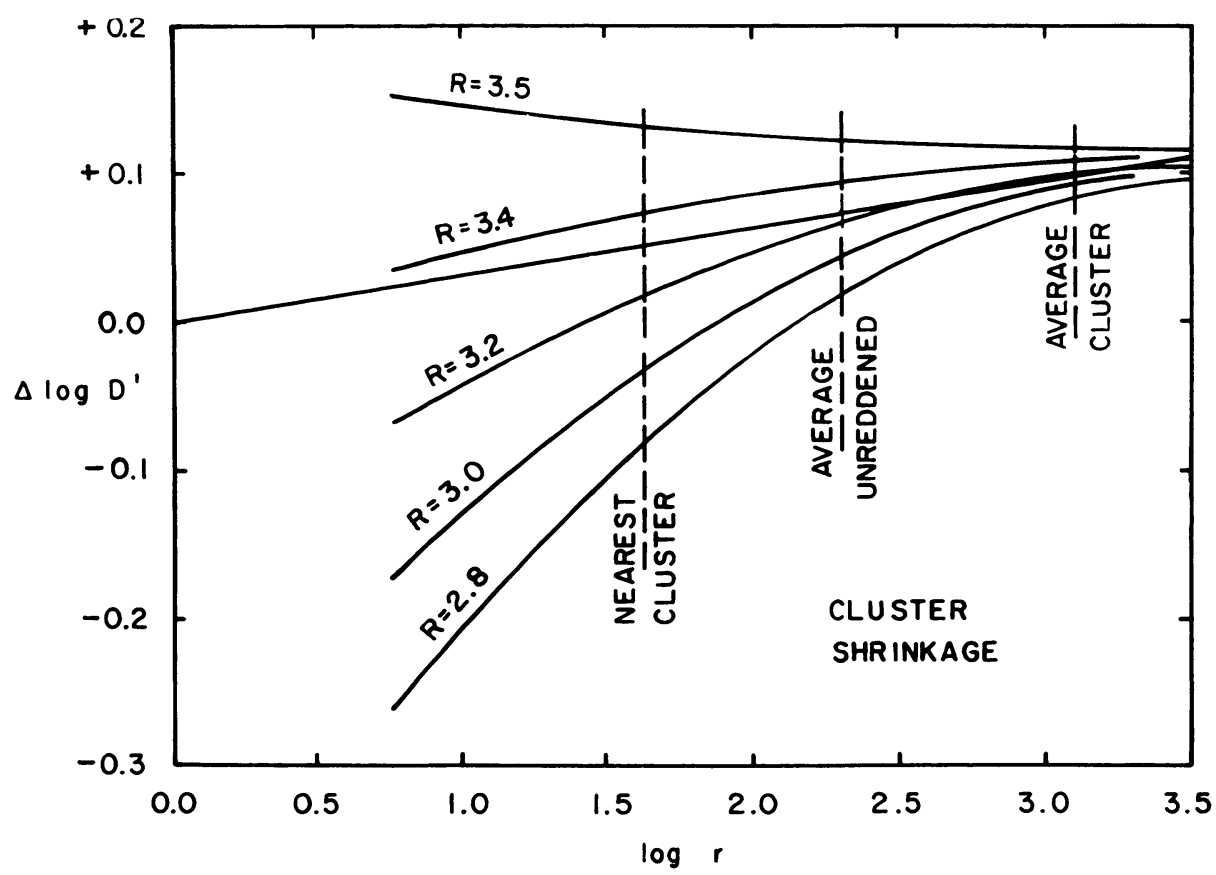

Fig. 11. The clusters shrinkage in going from unreddened to reddened clusters $\Delta \log D^{\prime}$ against distance for various $\mathbf{R}$ values. 
diameter relations presented in Figures 6,7 and 8 are good mean representations of the variation of apparent diameter with cluster distance.

Figure 10 presents the distance-color excess data for all clusters and $\mathbf{R}=3.2$. $A$ series of such plots for various $\mathbf{R}$ values shows that $E_{B-V}$ increases more linearly with distance for $\mathbf{R}$ values near 3.2. If $\mathbf{R} \simeq 3.2$ then $E_{B-V}(r)=(0.28 \pm 0.05 \mathrm{P}) \mathrm{mag}$. $/ \mathrm{kpe}$.

Figure 11 presents the distance dependence of the difference between the least squares fits to the reddened and unreddened clusters for various assumed $\mathbf{R}$ values. If each $\mathbf{R}$ value is in turn presumed correct then its curve represents the cluster shrinkage. The distance shown for the average unreddened cluster was computed using the mean reddening with distance given above. Note that for $\mathbf{R}>3.5$ the cluster shrinkage $\Delta \log D^{\prime}$ decreases with distance and $E_{B-V}$ while for $\mathbf{R}<2.8, \Delta \log D^{\prime}$ is negative for nearby clusters.

We will presume that clusters shrink slightly with reddening. This may be explained as the effect of extinction on the relatively faint stars near the cluster boundary. The diminished brightness of these stars reduces the apparent cluster diameter. If the cluster shrinkage is a smooth function of color excess only, then clusters can not expand with distance and reddening nor can nearby clusters be significantly larger than the mean unreddened clusters. Consequently we must have $2.8 \leq \bar{R} \leq 3.5$.

The shrinkage curves are uncertain by about 0.017 in $\Delta \log D^{\prime}$. Thus only the curves for $R \leq 3.0$ have significant curvature. Symmetrically between the limits in $\bar{R}$ is $\bar{R}=$ 3.15. A straight line fit to the curve for $\bar{R}=3.15$ seems the best representation for the shrinkage of all the clusters. If we can be confident at the ninety-five percent level that $2.8 \leq \bar{R} \leq 3.5$ and if the straight line is representative then $\bar{R}=3.15 \pm 0.20$. In this case, as color excess is linear with distance,

$$
\Delta \log D^{\prime} \simeq+0.127+0.044 \log E_{B-V}
$$

This means that $D^{\prime} \propto E_{B-V}{ }^{-0.044}$, indeed, a week dependence.

TABLE II

Final mean type residuals

\begin{tabular}{lllll}
\hline & I & II & III & IV \\
\hline$r$ & +0.148 & +0.148 & +0.289 & +0.40 \\
$m$ & -0.065 & +0.018 & +0.289 & +0.23 \\
$p$ & -0.190 & -0.190 & 0.0 & +0.15
\end{tabular}

Table II presents the final shrinkage corrected mean type residuals. Note that they are not much different from the preliminary values. Further work on the diameters of open clusters may prove of value to the study of interstellar grains and in the study of galactic structure. 


\section{References}

Becker, W.: 1963, Z. Astrophys. 57, 117.

Hagen, Gretchen L.: 1970, Publ. David Dunlap Obs. 4.

Johnson, H. L., Hoag, A. A., Iriarte, B., Mitchell, R. I., and Hallam, K. L.: 1961, Lowell Obs. Bull. 5, No. 113, 133.

Lynds, B. T.: 1967, Publ. Astron. Soc. Pacific 79, 448.

Trumpler, Robert J.: 1923, Publ. Allegheny Obs VI, 45.

Trumpler, Robert J.: 1930, Lick Obs. Bull. 14, No. 420, 154.

Wallenquist, Åke: 1959, Ann. Upp. Obs. 17, No. 7. 